\title{
LUCHA SILENCIOSA POR EL DERECHO (INDÍGENA) A LA CIUDAD: COMODATOS MAPUCHE EN EL A.M. DE SANTIAGO DE CHILE
}

\section{SILENT STRUGGLE FOR THE (INDIGENOUS) RIGHT TO THE CITY: MAPUCHE BAILMENTS IN THE METROPOLITAN AREA OF SANTIAGO DE CHILE}

Matthew Caulkins*

RESUMEN: Este artículo discute las complejidades del acceso de grupos indígenas a los espacios físicos, sociales y políticos de la ciudad. Es un acceso que conlleva complejidades toda vez que las ciudades han contribuido a la colonización de territorios indígenas en el pasado y siguen invisibilizando a aquellos sujetos indígenas migrantes residentes en sus áreas urbanas en el presente. El concepto lefebvriano de un derecho emergente, que nace de las luchas sociales en el espacio urbano, encuentra eco en los procesos vivenciados por asociaciones mapuche que acceden a terrenos entregados en comodato en el A.M. de Santiago. Estas asociaciones buscan practicar colectivamente la propiedad sobre esos espacios al llenarlos con actividades socialmente productivas, evitando así la rescisión de los contratos de comodato precarios. Es un activismo silencioso que se enfoca en ganar espacios concretos más que en movilizaciones políticas vistosas. Aquí se analiza la práctica social de la propiedad respecto a cinco temáticas: cómo los comodatos sirven como forma de gobernanza sobre las asociaciones; cómo tienen un impacto complejo en la autonomía de estos grupos; cómo son una performance; cómo buscan soporte en el espacio circundante y cómo presentan una tensa relación entre reivindicación moral y derechos legales. Por otro lado, esa práctica colectiva de la propiedad crea una pertenencia a los espacios del A.M. de Santiago que está en tensión con sus aspiraciones territoriales como pueblo en el sur del país. La discusión se basa en un proyecto de investigación cualitativo sobre luchas en torno a la propiedad, para lo cual se realizaron entrevistas con miembros de asociaciones indígenas coordinadoras de rukas en la metrópoli santiaguina.

Palabras clave: Derecho a la ciudad, Lucha Silenciosa, Práctica social de la propiedad, Pueblo Mapuche. 
ABSTRACT: This article discusses the contradictions involved in the access of indigenous peoples to physical, social, and political spaces of the city. These contradictions arise since cities have contributed to the colonization of Indigenous territories in the past and continue to make invisible those Indigenous individuals who migrate to, or reside within urban areas in the present. The Lefebvrian concept of an emergent right, which was forged through social struggles, finds echo in the processes experienced by Mapuche associations accessing urban land given in bailment in the metropolitan area of Santiago. These associations seek to collectively practice ownership of these spaces by filling them with socially productive activities, thus avoiding the termination of precarium bailment contracts. It is a silent activism that focuses on gaining concrete spaces rather than more visible political mobilizations. The social practice of property is analyzed here under five themes: how bailments serve as a form of governance over associations, how they have a complex impact on the autonomy of these groups, the performance of property, and property support in surrounding spaces and the tension between moral claims and legal rights. However, this collective practice of property creates a sense of belonging to the spaces of the metropolitan area of Santiago that is in tension with Mapuche territorial aspirations in the south of the country. The discussion is based on a qualitative research project on property struggles for which interviews were conducted with members of Indigenous associations that coordinate rukas (cultural centers) in Santiago.

Key words: Right to the city, Silent struggle, Social practice of property, Mapuche people.

Recibido: 27.07.19 Aceptado: 03.03.20

\section{INTRODUCCIÓN}

Este artículo indaga en la compleja relación de los pueblos originarios con las ciudades chilenas. Para eso utiliza el concepto lefebvriano del derecho a la ciudad para ahondar en la paradoja de la lucha de grupos indígenas por integrarse en áreas urbanas (Tomiak, 2011). La paradoja se refiere a dos aspectos, uno histórico y uno actual. La instalación de ciudades históricamente contribuyó a la colonización y pacificación de los territorios indígenas (Alvarado Lincopi, 2015). Por otro lado, en el imaginario urbano los sujetos indígenas estarían actualmente fuera de lugar, aunque estas mismas áreas sean sus tierras ancestrales. En efecto, aún persiste un imaginario de los pueblos indígenas como esencialmente rurales $y$, por lo tanto, no-urbanos. Asimismo, el racismo en las ciudades ha contribuido a invisibilizar a la población indígena presente ahí (Alvarado Lincopi, 2016). Estadísticas recientes indican que $15,5 \%$ de la población indígena urbana en Chile habla o entiende su lengua originaria mientras que en áreas rurales ese porcentaje es de un 25,8\% (Ministerio de Desarrollo Social, 2015). Pero no sólo eso, sino que también la vida urbana es una contradicción para el pensamiento actual de distintos sectores del pueblo mapuche. Estos sectores entienden a la ciudad como el espacio del colonizador del winka. Por lo tanto, no miran con buenos ojos a aquellos que han migrado a las áreas urbanas, porque el proyecto de recuperación territorial del pueblo mapuche se concentra en áreas rurales donde se concentran las reducciones históricas. 
Así también es la vida de los sujetos mapuche que han sido desplazados por el empobrecimiento forzoso de sus comunidades originales. Llegan a la metrópoli Santiaguina donde no tienen espacio para prácticas colectivas (Fontana \& Caulkins, 2016). Justamente es la experiencia anterior de los coordinadores de las asociaciones indígenas en organizaciones sociales en la lucha por la democracia (Antileo, 2007) que les ha dado a conocer la estrategia de requerir comodatos. Muchas son las municipalidades que entregan terrenos en comodato a organizaciones sociales. Pero es el Servicio de la Vivienda (SERVIU) del Ministerio de la Vivienda y Urbanismo la mayor institución comodante. Sólo en la Región Metropolitana SERVIU tiene un total de 532,4 hectáreas en comodato, ¡lo que constituye un impresionante $6,6 \%$ del área urbana de la región! (De acuerdo a datos de su Coordinador de Información Territorial, en 2012 el SERVIU era propietario de 20.714 hectáreas que correspondía a aproximadamente un cuarto de las 83.000 hectáreas del área urbana de Santiago para esa fecha.) Según entrevista con el jefe del departamento de gestión inmobiliaria del SERVIU, esos comodatos corresponden a terrenos asignados para equipamientos comunitarios que no se llegaron a construir en sus conjuntos de vivienda social. De acuerdo a archivos del SERVIU, revisados para el proyecto en septiembre de 2016 , sólo existen 5 terrenos en comodato con asociaciones indígenas (un total de 0,59 hectáreas), dos de los cuales fueron entrevistados para el proyecto (Parque Ceremonial Weichafe Mapu en Cerro Navia y ruka Folilche Aflaiai en Peñalolén).

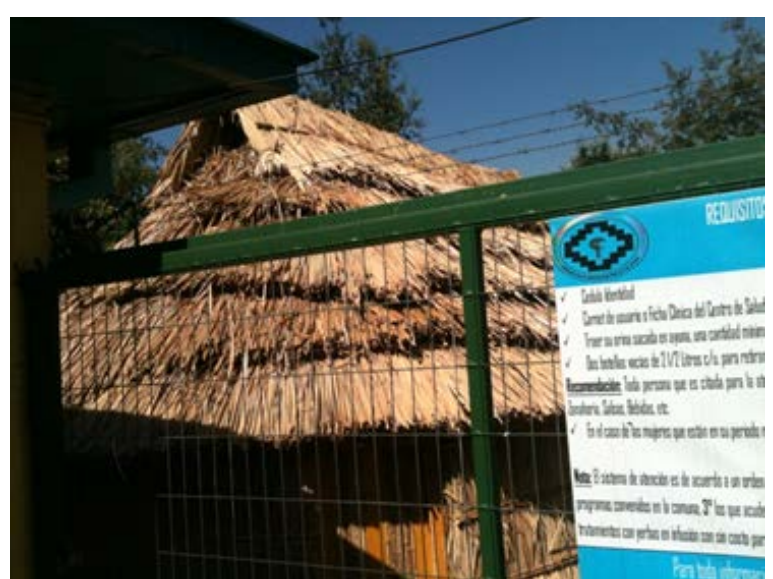

Figura 1. Ruka Kallfulikan en La Florida con aviso de atención de salud tradicional.

Fuente: foto del autor.

Es el interés por ahondar en esa contradicción que nos lleva a revisar a la geografía jurídica crítica en búsqueda de elementos para entender esa compleja relación con la tierra urbana. El argumento central de este artículo es que los comodatos constituyen una forma ambigua de acceder al derecho indígena a la ciudad. Propician tanto un acceso colectivo a los espacios físicos de la ciudad como un cierto control sobre los grupos mapuche por las instituciones comodantes (Figura 1). Permite de esa manera un acceso ambiguo a los espacios sociales y políticos de la ciudad.

\section{EL DERECHO A LA CIUDAD COMO PRÁCTICA DE LA PROPIEDAD}

El derecho a la ciudad fue concebido por Lefebvre en el aniversario del libro 'Das Kapital' de Marx. El concepto, originalmente propuesto en el turbulento año de 1968 en Paris, no es un derecho liberal de aquellos ofrecidos por el Estado. Ese derecho no se implementa sin lucha social y tal vez por eso ha sido adoptado como bandera de lucha por 
muchos movimientos sociales en las últimas décadas (Harvey, 2008). El derecho a la ciudad se construye por medio de la apropiación del espacio y su utilización para la autogestión de las camadas populares. El presente artículo propone que el concepto no ha perdido validez como estiman algunos (Yiftachel, 2009), sino que su valor está en colocar en evidencia las contradicciones inherentes que surgen entre las nuevas formas de sociabilidad urbana y derechos ciudadanos emergentes (Purcell, 2014). Es que el derecho a la ciudad lefebvriano no se puede entender $\sin$ el concepto complementario del derecho a la diferencia (Butler, 2012). Precisamente en los últimos años se ha utilizado el marco del derecho a la ciudad para analizar el acceso de las poblaciones indígenas a los espacios urbanos. Nioh (2017) explica como los grupos indígenas no tienen acceso a las tierras urbanas en Camerún por las formas que históricamente han tomado la urbanización y la tenencia del suelo. En Honolulu Grandinetti (2019) postula que el derecho a la ciudad podría implementarse trayendo el concepto tradicional de aloha 'āina (de la tierra como algo que necesita cuidados) hasta las ciudades donde prima actualmente el entendimiento de la tierra como mercancía en la renovación urbana. En islas Salomón, Foukona (2015) discute la estrategia utilizada por grupos indígenas locales de reivindicar legitimidad moral (un tema central del derecho a la ciudad) para acceder a la tierra urbana. Este artículo busca contribuir a esa literatura, indagando sobre la noción de derecho indígena a la ciudad y las complejidades de su incorporación en los regímenes propietarios locales.

La discusión entre ver el derecho a la ciudad como derecho legal o como reivindicación moral ha sido motivo de debates Huchzermeyer. 2018. Así, mientras algunos ven la incorporación del derecho a la ciudad en el Estatuto de la Ciudad brasilero, otros son críticos de los acomodos necesarios para su formalización en el aparato administrativo. Un análisis de la oeuvre de Lefebvre apunta a entender un rol provisorio para los derechos legales hacia su incorporación en prácticas sociales. ¿Entonces cómo podemos entender un derecho indígena a la ciudad? Attoh (2011) propone que los derechos pueden clasificarse en tres generaciones según su aparición en la historia. La primera generación serían las libertades políticas individuales, el derecho a un proceso justo. La segunda generación se refiere a los derechos socio-económicos, los resultados concretos. La tercera generación apunta a conceptos abstractos como culturas, idiomas y minorías. Aquí estarían los derechos de los pueblos a la autodeterminación recogidos en la determinación de las Naciones Unidas (Pairican, 2015). El pueblo mapuche busca justamente ese derecho a la autonomía como pueblo más allá de los derechos de primera o segunda generación, que son igualmente aspiraciones de los sujetos nacionales.

Sin embargo, una expresión más completa de un derecho indígena a la ciudad se enfrenta a dos visiones contrapuestas. Por un lado, el Estado busca encuadrar a los individuos indígenas urbanos en su visión de una economía de libre mercado. A partir del vuelco del activismo indígena con la vuelta a la democracia en 1990 y la visibilización de esa población en áreas urbanas con el Censo de 1992 (la primera en incluir pregunta sobre pertinencia étnica), los gobiernos de turno han ido creando una oferta de políticas para indígenas urbanos (Comisión Asesora sobre Política Indígena Urbana, 2006). Pero el enfoque siempre ha sido uno de apoyar el emprendimiento 
de individuos indígenas (Antileo, 2007). El mismo nombre de CONADI lo deja explícito: Corporación Nacional del Desarrollo Indígena. Por otro lado, para algunos sectores del pueblo mapuche la búsqueda del acceso a la ciudad contradice el proyecto del retorno al país mapuche (Alvarado Lincopi, 2015). Va a ser muy importante equilibrar el activismo político por la restitución de tierras en el territorio histórico mapuche con el derecho a la ciudad. Pero esa interrogante sobrepasa los límites del presente artículo, aquí buscamos entender lo que implica un derecho indígena a la ciudad.

Sin embargo, una expresión más completa de un derecho indígena a la ciudad se enfrenta a dos visiones contrapuestas. Por un lado, el Estado busca encuadrar a los individuos indígenas urbanos en su visión de una economía de libre mercado. A partir del vuelco del activismo indígena con la vuelta a la democracia en 1990 y la visibilización de esa población en áreas urbanas con el Censo de 1992 lla primera en incluir pregunta sobre pertinencia étnica), los gobiernos de turno han ido creando una oferta de políticas para indígenas urbanos (Comisión Asesora sobre Políitica Indígena Urbana, 2006). Pero el enfoque siempre ha sido uno de apoyar el emprendimiento de individuos indígenas (Antileo, 2007). El mismo nombre de CONADI lo deja explícito: Corporación Nacional del Desarrollo Indígena. Por otro lado, para algunos sectores del pueblo mapuche la búsqueda del acceso a la ciudad contradice el proyecto del retorno al país mapuche (Alvarado Lincopi, 2015). Va a ser muy importante equilibrar el activismo político por la restitución de tierras en el territorio histórico mapuche con el derecho a la ciudad. Pero esa interrogante sobrepasa los límites del presente artículo, aquí buscamos entender lo que implica un derecho indígena a la ciudad.

La propiedad es una forma de entender las relaciones de poder sobre los recursos. La geografía legal usualmente entiende la propiedad como un paquete de derechos. De lo cual desprende que podemos entender a la propiedad de dos formas principales. La propiedad privada liberal se refiere a la tenencia preferentemente individual del objeto suelo con el objetivo de facilitar la comercialización de terrenos entre extraños (Blomley, 2008). En esa visión las propiedades se simplifican para parecerse más a contratos, reduciendo sus ambigüedades para simplificar las transacciones comerciales. Al contrario, en este artículo entenderemos a la propiedad como práctica social. No existe una sola esencia de la propiedad (Blomley, 2008). Más bien interesa entender los efectos que se desprenden de ella. Blomley (2014) insiste que la propiedad no tiene una esencia a-histórica sino que nace de una performance. Por ejemplo, la puesta de una reja es una performance de la propiedad para dar a conocer al resto de la sociedad que ese espacio tiene un propietario.

Sarah Keenan (2014) conceptualiza la propiedad a través del pertenecer. Para eso extiende la idea del sentido común de que un objeto puede pertenecer a un sujeto lla base de la visión liberal de la propiedad) para incluir también la forma en que un sujeto pertenece a su ambiente. Ese sentido del pertenecer no existe independiente del espacio sino que recibe soporte de ese espacio circundante. Un sujeto blanco puede sentir que 'pertenece al espacio' de un mall en una comuna de altos ingresos. Sin embargo, puede sentir que 'no pertenece al espacio' en un campamento de bajos ingresos. Keenan (2014) argumenta que la propiedad depende del tiempo, ya que muchas veces 
dice respecto a situaciones temporarias como son los comodatos precarios. La propiedad, además, puede entenderse como una aspiración que se practica de forma pre-figurativa a la espera de que llegue a ser conforme al deseo del aspirante. En los asentamientos informales los sujetos actúan como propietarios: utilizando el espacio de forma exclusiva, construyendo viviendas $y / 0$ rejas aún ilegales, pero con la esperanza de que lleguen a ser reconocidos a futuro. Es también a esas discusiones sobre la práctica social de la propiedad que este artículo busca hacer un aporte. En este sentido, la práctica de la propiedad para grupos indígenas en áreas urbanas enfrenta distintos desafíos que los de los grupos indígenas en áreas consideradas no-urbanas y de otros grupos sociales en áreas urbanas.

Siguiendo esa lógica de la práctica social de la propiedad, los comodatos pueden entenderse como forma de disciplina sobre grupos marginalizados, según se desprende del análisis de Keenan (2013) sobre un conflicto en el Northern Territory de Australia. Al respcto, el gobierno federal australiano adquirió comodatos de forma compulsoria sobre los asentamientos informales donde reside la población indígena de la municipalidad de Alice Springs. El gobierno insistió siempre que su intención no era exigir la propiedad sobre esos espacios. Al contrario, utilizaron los comodatos así impuestos para imponer cambios en las condiciones de habitabilidad de las viviendas y en los comportamientos de la población local. Así, el derecho a la ciudad se refiere a un derecho por construir, una reivindicación moral donde prima la apropiación del espacio y su utilización en un programa de autogestión no estatal. Para entender su puesta en escena es importante distinguir entre una versión liberal de la propiedad y otra que se construye por medio de las prácticas sociales.

\section{MÉTODO}

Este artículo se basa en una investigación cualitativa enfocada en las asociaciones indígenas que coordinan rukas urbanas en Santiago. El trabajo de campo se realizó en el primer semestre de 2016. Fueron aproximadamente 3 meses de entrevistas semi-estructuradas donde se entrevistaron un total de 10 rukas o centros ceremoniales y un total de 18 entrevistados. Las rukas están ubicadas en Peñalolén (2), La Pintana (2), Cerro Navia (1), La Florida (1), Padre Hurtado (1), Puente Alto (1), Macul (1) y Recoleta (1) (Figura 2). Las preguntas se enfocaron en la trayectoria personal de los miembros o coordinadores de las asociaciones indígenas, las formas de tenencia de los terrenos, las formas de organización colectiva, las actividades que se realizan en esos espacios, los sentimientos de pertenencia al espacio circundante, la relación con el entorno, etc. También se buscó realizar entrevistas a funcionarios de las instituciones comodantes para los casos estudiados. Fueron entrevistados funcionarios de la Asesoría Urbana y de la Oficina Indígena de las municipalidades de La Pintana, Peñalolén y Cerro Navia. En La Pintana fue posible entrevistar también a funcionarios de la Secretaría de Planificación y de la Asesoría Jurídica. Además, se entrevistó a distintos funcionarios de CONADI y al director del departamento de gestión inmobiliaria del SERVIU-RM. El único comodante con el que no fue posible realizar una entrevista fue el Ministerio de Bienes Nacionales. La entrevista fue pospuesta por meses sin explicación alguna. El concepto que amarraba los 3 casos de estudio fue el de la práctica social de la propiedad o el 
reclamo por la propiedad que se practica de forma pre-figurada. El proyecto incluyó un caso sobre la ocupación de edificios abandonados en São Paulo y otro sobre el uso de espacios públicos en Melbourne. El concepto del derecho a la ciudad no era tan central en el proyecto como en este artículo. Pero aquí, al igual que en la tesis de doctorado de la investigadora candiense Julie-Ann Tomiak (2011) sobre las reservas indígenas urbanas en Toronto, se estimó que el concepto tiene validez explicativa aunque no haya formado explícitamente parte de las preguntas de las entrevistas.

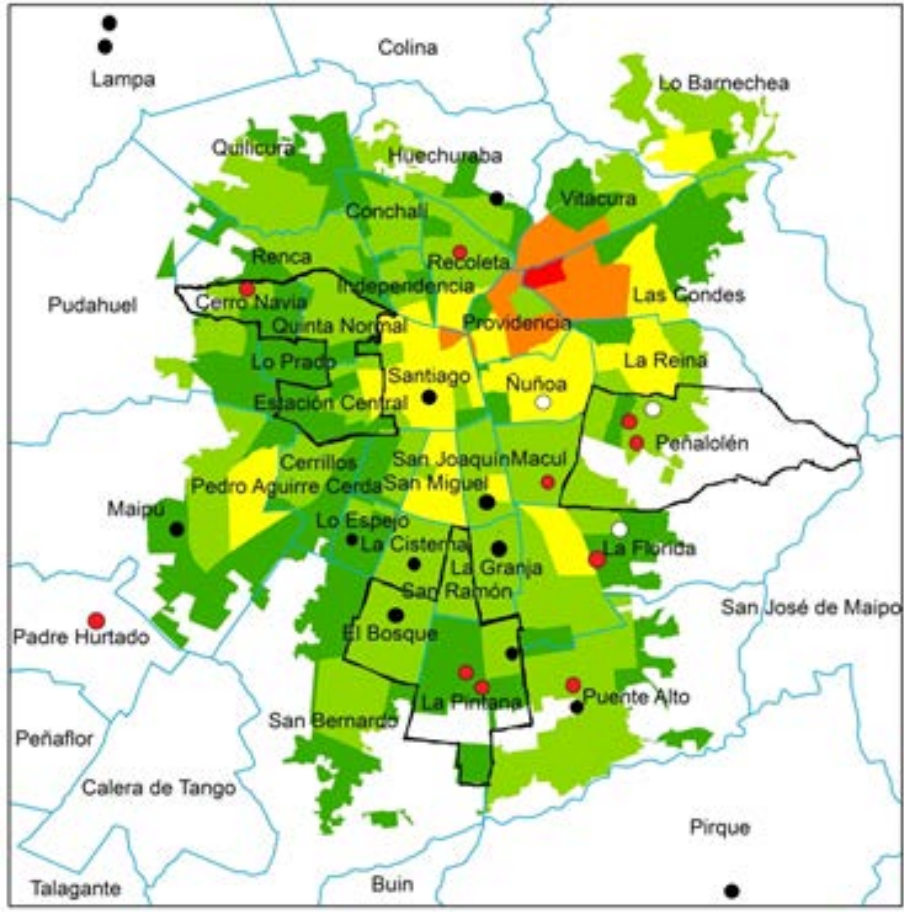

\section{Leyenda}

Poblacion Indigena

Comuna con $2.5 \%$ o

mas de poblasion

indegena segun dion

\section{Valores de suelo UF/m2}

Segun datos de X-Lab IEUT.PUC (2005)

$0,0 \cdot 2,1$

$2,2 \cdot 7,0$

$7,1 \cdot 16.5$

$16,6 \cdot 32,0$

$32,1 \cdot 71,5$

\section{Rukas}

Existentes -

Entrevistadas .

Abandonadas - 0

Figura 2. Valores de suelo, concentración de población indígena y ubicación de rukas. Elaborado por Nicolás del Canto

\section{DISCUSIÓN}

Históricamente migrantes mapuche han llegado a Santiago en búsqueda de mejores condiciones económicas. El acceso a un lugar en la ciudad para esta población indígena desplazada se da casi exclusivamente a través de la postulación a viviendas sociales y allegamiento con familiares (Fontana \& Caulkins, 2016). No hay acceso colectivo a la propiedad en la ciudad. La única forma de propiedad colectiva que existe en Chile es la propiedad indígena, y ésta por definición sólo existe en reducciones originalmente asignadas en áreas rurales. Incluso la restitución de tierras por CONADI no se da en el área metropolitana de Santiago a pesar del alto volumen de postulaciones que se hace en esa región (El Ciudadano, 2015). De todas maneras no sólo existen familias mapuche asentadas en Santiago, sino que existe la presencia del pueblo mapuche. 
Podemos notar esa presencia del pueblo mapuche en Santiago porque se organizan políticamente dentro de la urbe (Antileo, 2007) y reflexionan sobre sus experiencias urbanas (Alvarado Lincopi, 2015; 2016). En Santiago encontramos una variada construcción de espacialidades mapuche. El autor Mauro Fontana (2019) nota la creación de nuevas espacialidades urbanas mapuche a partir de cuatro tipologías espaciales: el aula intercultural de enseñanza del mapudungun, el lof de la Villa Bicentenario II, la ruka Mapu Lawen y la marcha mapuche que se hace cada 12 de octubre por la Alameda. Este artículo se enfoca específicamente en el caso de la entrega de espacios en comodato a asociaciones en rukas mapuche en la ciudad de Santiago (Sepúlveda \& Zúñiga, 2015). Pero éstos deben entenderse en el contexto más amplio de la construcción de un espacio físico, social y político para el pueblo mapuche en la metrópoli. Estos comodatos constituyen la ruta encontrada para el acceso colectivo a tierras urbanas para los grupos indígenas. El argumento aquí es que los comodatos constituyen una forma ambigua de acceder al derecho a la ciudad, siendo a la vez una forma de acceso a la ciudad y de control de las colectividades mapuche ejercido por las instituciones comodantes. Podemos entender un acceso a un derecho a la ciudad a través de la práctica de la propiedad en esos comodatos. Se analiza aquí cinco temáticas respecto de la práctica de la propiedad: gobernanza, impacto en la autonomía, performance, soporte en el espacio circundante y tensión entre reivindicación moral y derechos legales.

\section{FORMA DE GOBERNANZA}

Al pedir un comodato estas asociaciones no tienen la tenencia formal de esos terrenos. Cada contrato de comodato revisado empieza con un primer artículo que insiste que el SERVIU lo la municipalidad, si fuese el casol es el dueño del terreno. Ese artículo es importante porque se antepone a cualquier demanda de la entrega del terreno por usucapión /adquisición de la propiedad por su posesión continuadal al establecer que la asociación reconoce la propiedad de la institución comodante. Sin embargo, los coordinadores de las asociaciones en entrevista insistieron en su deseo de quedarse en los terrenos. Reconocen las limitaciones de la estrategia de los comodatos para encontrar terrenos urbanos para sus prácticas colectivas. Aunque quieren una mayor permanencia, saben que los comodatos sólo entregan una temporalidad prolongada en el tiempo.

Por eso podemos decir que los comodatos en Santiago exigen formas sutiles de disciplina a las asociaciones indígenas. Éstos se entregan solamente a aquellos grupos constituidos formalmente como asociaciones indígenas. Perpetúan la inseguridad de la tenencia al afirmar el poder unilateral del comodante de terminar el contrato a cualquier momento y por cualquier motivo. El contrato de comodato entre SERVIU-MINVU y la Asociación Folilche Aflaiai habla del 'derecho de poner término al contrato, en cualquier tiempo, cuando sobrevienen razones de oportunidad, mérito o conveniencia que justifiquen el ejercicio de la potestad revocatoria y así resulte necesario' lartículo sexto, Resolución Exenta 1223 de 6 de marzo de 20151. O como dicta el contrato de la Municipalidad de Macul con la Asociación Indígena Choyituyin Warria Mew, 'la Municipalidad podrá ponerle término en cualquier momento, sin derecho a indemnización de ninguna especie, previo aviso dado con 30 días corridos de anticipación' (Decreto Alcaldicio 1044 de 19 de agosto de 2014). 
Los comodatos se entregan de forma ad hoc a las organizaciones sociales que presenten un requerimiento formal a la institución comodante. El jefe del departamento de gestión inmobiliaria de SERVIU-RM explicó que las únicas exigencias explícitas son de entregarlos a organizaciones sin fines de lucro - ya que los comodatos son gratuitos - y que sean organizaciones formalmente constituidas, que tengan personalidad jurídica (llamada informalmente PJ). En el caso de los grupos mapuche se organizan y constituyen formalmente asociaciones indígenas para lograr la PJ tanto por sus menores exigencias como para figurar para distintos programas institucionales de CONADI.

Sin embargo, el departamento también trata de llevar en consideración una especie de utilidad social de esas organizaciones. Podríamos decir que el derecho a un lugar en la ciudad lo reciben aquellos que demuestran que se lo merecen. La idea es que los terrenos se entreguen a organizaciones que contribuyan /definido de manera amplia e implícita, esto es, no de forma explícita) a sus entornos sociales. Esa lógica la confirmó adicionalmente en entrevista el asesor jurídico de la municipalidad de La Pintana. Por eso las asociaciones necesitan demostrar una cierta productividad social para el comodante (municipalidad, SERVIU, etc.) para evitar la rescisión del contrato. Las asociaciones logran eso al llenar los espacios con una serie de actividades, como talleres de idioma, de orfebrería, conocimiento de hierbas nativas y salud ancestral.

Además, los contratos imponen otras condiciones al limitar los usos posibles en estos espacios. Primeramente, queda prohibida cualquier actividad que no conforma a las funciones descritas en el estatuto de la asociación. Esta no es una condición tan difícil de cumplir puesto que se incluyen ahí todas las "actividades culturales mapuche". Pero limita la libertad de que propongan actividades no explícitamente entendidas como indígenas. Quedan prohibidos usos residenciales. Esta condición se explica por la zonificación de los terrenos SERVIU como terrenos para equipamientos comunitarios (por lo tanto, no tienen función residencial). Muchos comentarios de los coordinadores explicaban las dificultades de proteger la seguridad de esos lugares sin poder dejar un cuidador viviendo en el local. Quedan prohibidas actividades que pudieran generar fondos económicos. Esta condición se explica por la entrega a costo cero de los terrenos a las asociaciones. Esto conlleva la dificultad de generar fondos para sus actividades, pues no tienen la libertad de hacer, por ejemplo, bingos.

\section{IMPACTO EN AUTONOMÍA}

Se distinguen dos distintos imaginarios políticos para el uso de la figura del comodato. Hay un imaginario de la propiedad oficial cuya 'función social' sería la de apoyar el buen funcionamiento del libre mercado (ver artículo 24, Constitución Política de la República de Chile). Hay otro imaginario contrario que busca contribuir a una continuidad del activismo mapuche en la ciudad, una conexión con la tierra (mapu) y la autonomía del pueblo mapuche (Pairican, 2015). El imaginario oficial insiste que el comodato es una figura de tenencia temporaria. Los contratos en general duran entre dos y tres años, a veces cinco. Pero, más allá de la duración del contrato, éstos dan al comodante la facultad unilateral de terminar el contrato en cualquier momento y por cualquier razón. Por otro lado, los mismos coordinadores de esos espacios aspiran a seguir de forma permanente en los 
terrenos. No desconocen las condiciones de los contratos de comodato. Buscan no dar ninguna excusa a los comodantes de rescindir los contratos. Esa aspiración de permanencia encuentra soporte en una serie de actividades que realizan para mantener llenos esos espacios preferentemente durante toda la semana.

Las actividades a la vez sirven para cumplir con la condiciones de la gobernanza y como apoyo a la autonomía. Una mayor autonomía se sugiere al denominar a todas las actividades que no conllevan financiamiento estatal de 'autogestionadas' mientras que las actividades financiadas son justamente aquellas que valora la entidad financiadora. Muchos de las actividades reciben distintos fondos estatales (Fondart, PESPI, Fondo Nacional de Desarrollo Regional, etc.) para su ejecución. Las asociaciones pueden postular a esos fondos precisamente porque tienen dirección institucional dado por el comodato. Esas actividades financiadas mantienen los espacios llenos durante los días de semana - cuando de otro modo estarían vacíosMuchos de los participantes trabajan en otros oficios o profesiones durante la semana y sólo con la remuneración es que pueden hacer el tiempo para ofrecer esos talleres en horario de semana. El programa especial de salud y pueblos indígenas (conocido como PESPI) es la más importante en términos económicos, donde las asociaciones contribuyen justamente a complementar a la debilitada red de salud pública de sus entornos con atención de machi o con talleres realizadas por miembros de las asociaciones.

Solamente se encontró un caso donde la asociación estuvo a punto de negociar la formalización de la propiedad. Según comentario del coordinador Raimundo Nahuel, de la asociación Folilche Aflaiai de Peñalolén, estuvieron en negociaciones para la transferencia del terreno. Cuando preguntado, el representante de SERVIU explicó que no pueden hacer transferencia de terrenos porque no está dentro de la definición de las funciones de la institución. Esa lógica tiene poca credibilidad, ya que ¡tampoco tienen dentro de sus funciones ser dueños de gran cantidad de terrenos en la Región Metropolitana! En el fondo, el funcionario solamente dejaba en claro que las transferencias sólo se pueden lograr a través de medidas excepcionales. Eso concuerda con la información entregada por Raimundo, quien insistió en la necesidad de un apoyo político para esta transferencia, por lo que menciona:

"La idea ahora, en un momento dado, nosotros estuvimos haciendo las gestiones, llegamos hasta el momento de que era voluntad política, del traspaso, que fue antes que terminara el [primero] gobierno de Piñera ... cuando Piñera se iba a ir habían unas personas que dijeron no, aquí hay pura voluntad política, cierto, y la voluntad política no llegó nunca. Dijeron que teníamos que tener algún contacto con algún senador ahí"

\section{PERFORMANCE}

El comodato permite una entrega temporaria de la tenencia de la tierra urbana a las organizaciones sociales. Las rukas construidas en esos comodatos contribuyen a atraer la población mapuche local a actividades socioculturales como palin, mingako, clases de cosmovisión - de lengua mapudungun, orfebrería, medicina tradicional, atención de machi, etc. Otra forma en que visibilizan a la cultura mapuche es con la realización de ceremonias espirituales colectivas. En muchos de esos comodatos se realizan 
el nguillatun (año nuevo mapuche). En muchos terrenos en comodato, algunos con ruka, otros sin ruka, se han plantado rewes (altares) donde se congregan grandes cantidades de vecinos para la realización de esas ceremonias. Son ceremonias que funcionan para celebrar la cultura mapuche, pero también para acercar a gente de familias mapuche que tal vez no tenían tan presente sus raíces.

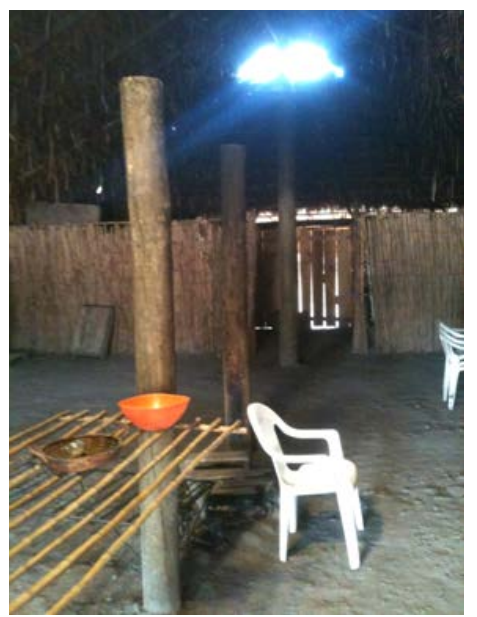

Figura 3. Interior de ruka Mapu Rayen en Padre Hurtado. Fuente: Foto del autor.

Por lo tanto, en ambos casos lo que está en juego es una performance de lo indígena o de lo auténticamente originario. Taiaiake Alfred \& Jeff Corntassel (2005 citado en Gilbert 2013, p. 174, traducción nuestra) definen la performance de lo indígena como "pensar, hablar y actuar con la intención consciente de regenerar la indigeneidad de uno mismo". Como afirman Véliz, Cerna \& Samit-Orniz (2019) respecto a las comunidades indígenas en el norte de Chile, "la propiedad raíz, entendida como una red de relaciones corporativas y alterizadas, es un elemento estructurador y performativo de la comunidad (...) indígena actual". De igual manera, las rukas presentan una marcada materialidad 'originaria', la mayoría son de madera con techo de paja y con piso de tierra (Figura 3). De esa forma, se presentan como un auténtico espacio indígena. Para muchos de los visitantes a las rukas esa materialidad 'originaria', la mayoría son de madera con techo de paja y con piso de tierra (Figura 3). De esa forma, se presentan como un auténtico espacio indígena. Para muchos de los visitantes a las rukas esa materialidad los remite a los espacios más rurales del sur. También la espacialidad trae a la ciudad estas formas auténticamente indígenas. Los entrevistados mencionaban la importancia de la orientación proporcionando la entrada del sol en una entrada por la mañana y la salida por la entrada opuesta en la tarde. Varios hacían hincapié en la importancia del fuego para curar a la techumbre y ahuyentar a las arañas. Muchas entrevistas fueron acompañadas de fuego, otros con intentos fallidos de mantener el fuego y de concentrar en el intercambio artificial de una entrevista semi-estructurada. Sin embargo los que han tenido que cambiar alguna de estos elementos son criticados por una supuesta falta de autenticidad. La ruka Mapu Lawen en La Pintana tuvo que poner piso de cerámica como exigencia del programa de salud (PESPI) con que trabajan. La performance de la autenticidad es tanto fuente de poder sobre el espacio como de contradicciones en el trabajo de practicar la propiedad sobre estos espacios.

El coordinador Raimundo explicó, además, la importancia de recibir a estudiantes tanto de la educación secundaria como de las universidades, tanto nacionales como extranjeras, y apoyarlos en sus investigaciones, tesis y entrevistas sobre el pueblo mapuche. Es una performance de embajadores apoyando la difusión de una cultura extranjera a la metrópoli. Todas estas actividades sirven a la vez para dar visibilidad al pueblo mapuche en la ciudad y así dan mayor percepción de seguridad de tenencia a estas agrupaciones. 


\section{SOPORTE EN ESPACIO CIRCUNDANTE}

Todas las asociaciones entrevistadas para esta investigación insistieron que los terrenos antes de su llegada estaban abandonados. Eran micro-basurales, llenos de basura y muchas veces foco de delincuencia. Al buscar terrenos desocupados y darles una nueva vida socialmente productiva, las asociaciones están demostrando un cuidado por la tierra muy distinto al cuidado oficial hacia esas mismas tierras.

En el imaginario oficial los sitios eriazos son señal de baja productividad económica para algunos gobiernos (como en programas que incentivan el uso de terrenos y edificios abandonados en el plan regulador intercomunal de São Paulo de 2014) y externalidades necesarias al buen funcionamiento del mercado para otros. Los gobiernos simplemente no tienen la capacidad ni la preocupación de cuidar de los sitios eriazos de su propia propiedad. La magnitud de terrenos del SERVIU bajo comodato lo deja claro. Repite la políitica del Estado chileno de declarar las tierras al sur del Biobío vacías e improductivas en el siglo XIX. Esas tierras eran muy productivas y estaban llenas de actividades sociales y espirituales. Pero fue el imaginario político de la propiedad liberal que impedía al gobierno verlo, ya que esperaban ver actividades económicamente productivas que se pareciesen a la agricultura tradicional europea.

Aunque el jefe de departamento de gestión inmobiliaria de SERVIU insistió en la imposibilidad del traspaso de terrenos a organizaciones sociales comodatarias, toda explicación simplista sobre lo que 'es' la propiedad se pierde rápidamente en ofuscaciones más que en explicaciones pertinentes. Las propiedades formales se pueden transferir de muchas formas. Las ocupaciones ilegales de terrenos se pueden transformar en propiedad formal a través de la figura de la usucapión. En todo caso, los comodatos no llegan a ese extremo. Más bien buscan una figura legal que sí encuentra, como diría Keenan (2014), un pertenecer con soporte en el espacio político circundante. Como dijo la coordinadora Beatriz Painiqueo, también de la ruka Folilche Aflaiai, sobre la posibilidad de hacer toma de terreno en un área urbana:

Obviamente toma no podemos hacer, porque aquí lo que más prevalece, es simplemente lo que es la represión, a través de la policía, entonces obviamente hay que tener tiempo, dedicación y todo, para hacer tomas, entonces eso era inalcanzable. Por lo tanto, la opción es tocar puertas, solicitar, mandar cartas e ir.

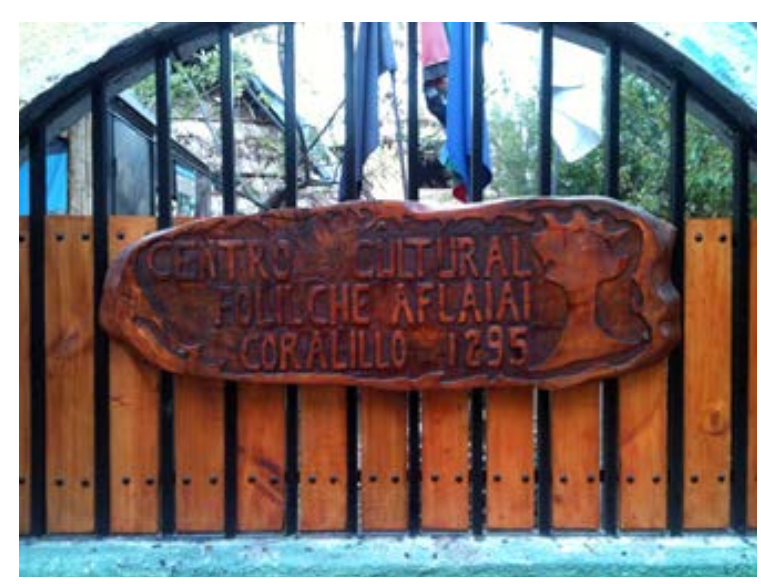

Figura 4. Acceso exterior a Ruka Folilche Aflaiai. Fuente: foto del autor.

Las asociaciones no sólo buscan que su inserción urbana pase 'bajo el radar' (Bayat, 2000), sino además se preocupan de hacerlo de la forma más legal posible para evitar posibles repercusiones como la rescisión de los contratos de comodato (Figura 4). 
Las asociaciones no sólo buscan que su inserción urbana pase 'bajo el radar' (Bayat, 2000), sino además se preocupan de hacerlo de la forma más legal posible para evitar posibles repercusiones como la rescisión de los contratos de comodato (Figura 4).

Pero su simple presencia año tras año ha logrado hacer cambios en el espacio social circundante. Al principio la relación con los vecinos no era buena. Como dijo el miembro de la asociación Folilche Aflaiai Pablo Ancatén, 'los primeros cuatro años, amenaza de que nos iban a quemar el sitio, que nos iban a quemar una casa que teníamos antes acá'. Pero con el tiempo esas relaciones se fueron cambiando. Él cree que un factor esencial en ese cambio estaba en la atención de machi que se hace en la ruka, 'creo que lo que cambió la gran vista de todo... fue la atención de machi... empezaron a venir gente y empezaron a sanarse'. El mismo participante explica otras actividades que se hicieron para mejorar las relaciones vecinales.

"de repente hacíamos cine, cine - documental abierto, sacábamos un telón y ponía, proyectamos eh, películas pa' los niños, documentales, después vendíamos sopaipillas; no, de primera regalábamos sopaipillas y café de trigo"

\section{TENSIÓNENTREREIVINDICACIÓN LEGAL Y MORAL}

La discusión aquí ha querido resaltar una especie de derecho emergente a la ciudad que está en construcción por el pueblo mapuche en el A.M. de Santiago. Pero es importante no confundir esa reivindicación moral con derechos legales, ya que esos todavía no se han conquistado. El disciplinamiento ejercido por la figura del comodato se hizo notar recientemente cuando SERVIU rescindió el contrato de la asociación indígena CONACIN al espacio superior del Cerro Huechuraba lo Cerro Blanco). Sin embargo, también estuvo presente el quiebre entre imaginarios legales. Los integrantes de la asociación indígena - compuesta de miembros de distintos pueblos - se rehusaron a dejar el local, que insisten, les pertenece. Además, insisten en sus derechos según el convenio 169 de la Organización Internacional del Trabajo. Hasta el momento el caso sigue abierto, pero el SERVIU parece intuir la profundidad de la diferencia entre mundos normativos. En 2016 traspasó el comodato, junto con el problema, a la municipalidad de Recoleta (Massai, 2019). Ésta ha reclamado que la permanencia de la asociación impide el derecho de otra asociación indígena que quisiera utilizar el espacio. Pero la municipalidad no ha querido activar la fuerza de la ley para obligarlos a dejar el lugar del comodato rescindido.

Otro caso también ilustra la tensión entre reivindicación moral y derecho legal. Una asociación mapuche de Lo Prado demandó a la empresa Transelec S.A. por vicisitudes en la consulta indígena del Estudio de Impacto Ambiental (EIA) del proyecto de instalación de una subestación eléctrica del metro (Arias, 2013). Alegaban que la subestación impedía la comunicación de la machi con los seres espirituales a través del rewe en el terreno vecino entregado en comodato por la Municipalidad de Lo Prado a la asociación indígena. La jueza de la séptima sala de la corte de apelaciones de Santiago falló en su contra estimando que ya se había realizado una consulta indígena y eso era suficiente. (El caso es Sebastián Collonao Marilao y Rosa Cayún Chanqueo vs. Comisión de Evaluación Ambiental Región Metropolitana, octubre 2014, Séptima Sala de 
la Corte de Apelaciones de Santiago, Recurso de Protección.) Sin embargo, con eso confirmó de manera implícita el derecho de la asociación a la primera consulta indígena (Ugarte, Fontana \& Caulkins, 20191. Lo significativo de ese gesto es que aunque no son propietarios del terreno, según la visión liberal, sino sólo comodatarios. El caso demuestra un derecho emergente a partir de la práctica de una forma temporaria de propiedad y su impacto incipiente en los derechos legales.

\section{CONCLUSIONES}

En este artículo se ha revisado la estrategia que han ocupado agrupaciones mapuche para encontrar espacios para sus prácticas colectivas en la ciudad de Santiago. La ruta de los comodatos sirve paradójicamente para conquistar el derecho indígena a la ciudad, pero no a la propiedad privada. A partir del análisis de este caso, el derecho (indígena) a la ciudad se entiende como una estrategia ambigua, tanto de acceso a los espacios físicos, sociales y políticos de la ciudad como de gobernanza ejercida por las instituciones comodantes. Se analizó como los comodatos se pueden entender como práctica social de la propiedad a través de cinco temáticas: gobernanza, impacto en la autonomía, performance, soporte en el espacio circundante y tensión entre reivindicación moral y derechos legales. Además, se analizó cómo ese derecho está en tensión con el proyecto territorial del pueblo mapuche en el sur del país. Aquí se insiste que el derecho a la ciudad se está conquistando por una invasión silenciosa (Bayat, 2000) y no de forma más abiertamente conflictiva como en otras latitudes. A la vez el caso de los comodatos mapuche en Santiago nos da un entendimiento renovado del concepto lefebvriano de derecho a la ciudad. Este entendimiento nos permite comprender las estrategias indígenas de buscar acceso a los espacios de las ciudades. Este artículo ha argumentado que es un activismo basado en la apropiación del espacio que busca promover en última instancia mayor autodeterminación para el pueblo mapuche.

\section{FINANCIAMIENTO}

El presente trabajo ha sido financiado por el Australian Research Council [DP140102851].

\section{AGRADECIMIENTOS}

Se agradece la contribución del Dr. Mauro Fontana en discusiones tempranas respecto a la confección del artículo y los comentarios constructivos de los revisores anónimos que ayudaron a dejar más claro los resultados de esta investigación. 


\section{REFERENCIAS BIBLOGRÁFICAS}

Alvarado Lincopi, C. (2015). La emergencia de la ciudad colonial en Ngülu Mapu: control social, desposesión e imaginarios urbanos. En E. Antileo, L. Cárcamo-Huechante, M. Calfío, y H. Huinca-Puitrin (Ed.), Awükan ka kuxankan zugu wajmapu mew. Violencias coloniales en Wajmapu (107-139). Temuco: Ediciones Comunidad de Historia Mapuche.

Alvarado Lincopi, C. (2016). Silencios coloniales, silencios micropolíticos. Memorias de violencias y dignidades mapuche en Santiago de Chile. Aletheia, 6(12), 1-17. Recuperado de http://sedici. unlp.edu.ar/handle/10915/53995

Antileo, E. (2007). Mapuche y santiaguino: El movimiento mapuche en torno al dilema de la urbanidad. Nuke Mapuforlaguet. En J. Calbucura (Ed.), Working Paper Series 29. Recuperado de http://www.mapuche. info/wps_pdf/antileo070700.pdf

Arias, F. (2013). La pelea de los mapuche de Lo Prado. The Clinic. Recuperado de https://www.theclinic.cl/2013/06/14/ la-pelea-de-los-mapuche-de-lo-prado/

Attoh, K. A (2011). What kind of right is the right to the city? Progress in Human Geography, 35(5), 669-685. doi: $10.1177 / 0309132510394706$

Bayat, A. (2000). From 'Dangerous Classes' to 'Quiet Rebels': Politics of the Urban Subaltern in the Global South. International Sociology, 15(3), 533-557. doi: $10.1177 / 026858000015003005$

Blomley, N. (2008). Enclosure, Common Right and the Property of the Poor. Social \& Legal Studies, 17(3), 311-331. doi: $10.1177 / 0964663908093966$
Blomley, N. (2014). Learning from Larry: Pragmatism and the Habits of Legal Space. En I. Braverman, N. Blomley, D. Delaney y A. Kedar (Eds.), The Expanding Spaces of Law: A Timely Legal Geography 177 . 94). Stanford, CA: Stanford University Press.

Blomley, N. (2016). Book Review: Subversive Property: Law and the Production of Spaces of Belonging. Social \& Legal Studies, 25(4), 509-51 1. doi: $10.1177 / 0964663916647530$

Butler, C (2012). Spatial Politics, Everyday Life and the Right to the City. Abingdon: Routledge.

Comisión Asesora sobre política indígena urbana. (2006). Propuesta para la generación participativa de una política indígena urbana: Informe de la Comisión Asesora. Santiago: Ministerio de Planificación. Recuperado de http:// www. desarrollosocialyfamilia.gob.cl/ btca/txtcompleto/polit.indigenasurbanos. pdf

El Ciudadano. (2015). Denuncia fraude y engaño en el concurso de subsidio de adquisición de Tierras de Conadi en la Región Metropolitana. Recuperado de https://www.elciudadano.com/ politica/denuncia-fraude-y-engano-enel-concurso-de-subsidio-de-adquisicionde-tierras-de-conadi-en-la-regionmetropolitana/05/05/

Fontana, M. (2019). Wariatun, Espacialidades mapuche en el Área Metropolitana de Santiago. Caso: Desplazamiento mapuche hacia el Área Metropolitana de Santiago 1975 2016 (Tesis doctoral). Universidad Católica de Chile, Santiago. 
Fontana, M., y Caulkins, M. (2016). Espacios Mapuche en el Área Metropolitana de Santiago Hoy: Paradojas sobre la propiedad y el territorio. Revista Planeo, 28, 1-12. Recuperado de http://revistaplaneo. uc.cl/wp-content/uploads/ Arti\%CC\%8 1 culo_FontanaCaulkins.pdf

Foukona, J. (2015). Urban Land in Honiara: Strategies and Rights to the City. The Journal of Pacific History, 50(4), 504-518. doi:10.1080/00223344.2 015.1110328

Gilbert, H. (2013). Indigeneity and Performance. Interventions, 15(2), 173-180. doi: $10.1080 / 1369801 \times .2013 .798467$

Grandinetti, T. (2019). Urban aloha 'aina: Kaka'ako and a decolonized right to the city. Settler Colonial Studies, 9(2), 227-246. doi: $10.1080 / 2201473 \times .2017 .1409400$

Harvey, D. (2008). The Right to the City. New Left Review, 1531, 23-40.

Huchzermeyer, M. (2018). The legal meaning of Lefebvre's the right to the city: addressing the gap between global campaign and scholarly debate. Geolournal, 83(3), $631-644$ doi: 10.1007/s $10708-017-9790-y$

Keenan, S (2014). Subversive Property: Law and the Production of Spaces of Belonging. New York: Routledge.

Keenan, S. (2013). Property as Governance: Time, Space and Belonging in Australia's Northern Territory Intervention. The Modern Law Review, 76(3), 464-493. doi: 10.1111/14682230.12021
Massai, N. (2019). El conflicto por el Cerro Blanco que salpica al alcalde Jadue. Interferencia. Recuperado dehttps://interferencia.cl/articulos/elconflicto-por-el-cerro-blanco-que-salpicaal-alcalde-jadue

Ministerio de Desarrollo Social. (2015). Pueblos Indígenas: Síntesis de Resultados. Encuesta de Caracterización Socioeconómica Nacional CASEN-2015. Santiago. Recuperado de http://observatorio. ministeriodesarrollosocial.gob.cl/ casen-multidimensional/casen/docs/ CASEN_2015_Resultados_pueblos_ indigenas.pdf

Nioh, A. J. (2017). "The Right-ToThe-City Question" and Indigenous Urban Populations in Capital Cities in Cameroon. Journal of Asian and African Studies, 52(2), 188-200. doi: $10.1177 / 0021909615570954$

Pairican, F. (2015). El retorno de un viejo actor político: El guerrero. Perspectivas para comprender la violencia política en el movimiento mapuche (1990-2010). En E. Antileo, L. Cárcamo-Huechante, M. Calfío, y H. Huinca-Puitrin (Eds.), Awükan kuxankan zugu kiñeke rakizuam. Violencias coloniales en Wajmapu (pp. 301-324). Temuco: Ediciones Comunidad de Historia Mapuche.

Purcell, M. (2014). Possible Worlds: Henri Lefebvre and the Right to the City. Journal of Urban Affairs, 36(1), 141154. doi: 10.1111/juaf. 12034

Sepúlveda, B., \& Zúñiga, P. (2015). Geografías indígenas urbanas: el caso mapuche en La Pintana, Santiago de Chile. Revista de Geografía Norte Grande, (62), 127-149. 
Recuperado de https://scielo.conicyt.cl/ Colonial Studies, 9(2), 187-206. doi: scielo. php? script=sci_arttext\&pid=S0718- 10.1080/2201473X.2017.1409397 $34022015000300008 \& n r m=i s o$

Véliz, L., Cerna, C., \& Samit-Oroz, S. Tomiak, J.-A. (2011). Indigenous selfdetermination, neoliberalization, and the right to the city: Rescaling aboriginal governance in Ottawa and Winnipeg (Tesis doctoral). Carleton University, Ottawa.

Ugarte, M., Fontana, M., \& Caulkins, M. (2019). Urbanisation and Yiftachel, O. (2009). Critical theory Indigenous dispossession: rethinking and 'gray space': Mobilization of the the spatio-legal imaginary in Chile colonized. City, 13(2-3), 246-263. doi: vis-à-vis the Mapuche nation. Settler 10.1080/13604810902982227 